\title{
ESCALAS DE MEDO ODONTOLÓGICO EM CRIANÇAS E ADOLESCENTES: UMA REVISÃO DE LITERATURA ${ }^{1}$
}

\author{
Marcela Maia Tambellini \\ FFCLRP - Universidade de São Paulo \\ Ricardo Gorayeb ${ }^{2}$ \\ FMRP - Universidade de São Paulo
}

\begin{abstract}
Resumo: O presente artigo traz uma análise feita a partir de uma revisão da literatura sobre escalas que quantificam ou classificam o medo em crianças e adolescentes quando eles são submetidos a tratamento odontológico. A seleção da bibliografia avaliada incluiu artigos e livros, focalizando o período de 1900 a 2001, dirigidos a temas como medo e ansiedade. Os resultados mostraram inicialmente que a maioria das escalas encontradas era direcionada a escolares, sendo poucos os artigos que relatavam trabalhos com escalas para pacientes abaixo de cinco anos. Observou-se também que a maioria das escalas consistia em uma adaptação de outras, geralmente destinadas a adultos. Esses dados levaram à identificação da necessidade de se elaborar uma nova escala, dirigida à avaliação de crianças de um a três anos, passível de ser utilizada pelo odontopediatra, que recebeu o nome de Escala de Observação do Comportamento Infantil.
\end{abstract}

Palavras-chave: Escalas; medo; odontologia.

\section{ODONTOLOGICAL FEAR SCALES IN CHILDREN AND ADOLESCENTS: A LITERATURE REVIEW}

\begin{abstract}
This paper presents an analysis conducted through a literature review about scales that quantify or classify fear in children and teenagers, when submitted to dental treatment. The bibliography selection included papers and books from the $20^{\text {th }}$ century, especially those about fears and anxieties. The results initially showed that most of the scales found were made for school children, with few articles about patients below 5 years old. It was also noticed that the majority of scales consisted of adaptations of others, generally made for adults. Therefore, the data showed that there was a need for the construction of a new scale, for children aged 1 to 3 years old, which could be used by pediatric dentists, and that scale was named Scale of Children Behavior Observation
\end{abstract}

Key-words: Scales; fear; odontology

Na Odontopediatria a pronta aceitação da experiência odontológica para crianças antes de 4 anos de idade é difícil, pois a boca é uma região muito sensível e íntima, e nesta fase elas têm certa dificuldade de comunicação, são dependentes dos pais física e emocionalmente e costumam manifestar medo ante o inesperado e o desconhecido (Silva, Ammon \& Vieira, 1994). Segundo Guedes-Pinto (1991), durante a fase que vai até 1 ano de idade, quando a

\footnotetext{
${ }^{1}$ Artigo recebido para publicação em 07/03/03; aceito em 23/07/03.

${ }^{2}$ Endereço para correspondência: Ricardo Gorayeb, Departamento de Neurologia, Psiquiatria e Psicologia Médica, Faculdade de Medicina de Ribeirão Preto, USP, Av. Bandeirantes, 3900, Monte Alegre, Ribeirão Preto, SP, Cep 14049-900, E-mail: rgorayeb@fmrp.usp.br
}

boca é o principal meio de relação com o mundo, a personalidade da pessoa está sendo formada na sua quase totalidade, portanto mexer com a boca, significa mexer com o esquema mais antigo e conhecido de vida de cada indivíduo. Para este trabalho, (Alwin, Murray \& Niven, 1994; Hosey \& Blinkhorn, 1995; Ten Berge, Hoogstraten, Veerkamp \& Prins, 1998) o profissional indicado é o odontopediatra com experiência e não somente com o título de especialista. Como colocam Frankl, Shiere e Fogels (1962), a idade pré-escolar é um período que compreende um desenvolvimento emocional e físico imenso, havendo aumento do medo, e, em uma sala de atendimen- 
to, encontram-se barulhos, movimentos inesperados, dor, pessoas novas e imagens ou cheiros estranhos. A criança deve aprender a lidar com estes medos, caso contrário irá reagir: chorando, chutando, ou "lutando". Efetuou-se uma análise crítica da literatura nesta área, para identificar necessidades de pesquisa.

\section{Revisão de Literatura}

A revisão de literatura teve como foco principal temas que estavam relacionados ao estudo do comportamento de crianças, escolares, pré-escolares e adolescentes, durante o atendimento odontológico. Buscaram-se artigos e livros que versavam sobre um tópico comum: o medo e/ou ansiedade relacionados ao ambiente profissional e ao tratamento odontológico. A pesquisa foi realizada na internet, utilizando-se do sistema Pubmed e Lilacs, no período de 1900 até 2001, buscando selecionar artigos de odontologia e de psicologia. Os artigos mais antigos encontrados sobre este tema situaramse no final da década de 50 e início da de 60. A maioria dos autores quantificava medo e ansiedade e dividia as formas de avaliação em questionários e escalas.

Os questionários eram aplicados em adultos, adolescentes e escolares e as escalas em pré-escolares. A justificativa dos autores era de que o questionário exigia maior compreensão e cooperação por parte do sujeito analisado e as escalas eram simples e objetivas, fáceis de serem aplicadas e não dependiam tanto da cooperação do paciente.

Uma revisão da literatura realizada por Winer (1982) mostrou que a análise das reações das crianças às situações em consultório odontológico constituiu-se em uma área da pesquisa com grande expansão em poucos anos, e com isso, surgiram inúmeros artigos que envolviam crianças de diferentes idades, status social e que abrangiam uma gama imensa de medidas e variáveis. O seu trabalho tinha como objetivo informar sobre as novas descobertas, apresentar algumas generalidades a respeito de comportamento e origens do medo e estimular um estudo adicional com espírito crítico e interpretativo.

Os artigos que apareceram depois traziam resultados de pesquisas empíricas com aplicações de escalas ou questionários e coleta de dados, em dife- rentes idades, classes sociais, níveis de escolarização, alguns aplicados nos pais e outros à própria criança.

Dois são de fundamental importância para quem quer saber a origem das escalas de avaliação de ansiedade; o primeiro deles é o de Frankl e cols (1962), que, além de realizar uma revisão de literatura, criou uma escala de avaliação de comportamento de pré-escolares, simples e objetiva. Porém, um marco de referência para todos os artigos posteriores analisados foi o trabalho de Corah (1969), que criou uma escala para medir ansiedade, cuja importância foi evidenciada porque a mesma era simples, objetiva e de fácil aplicação, além de válida e confiável, consistindo de quatro perguntas com cinco possíveis respostas, sendo que somente uma deveria ser assinalada e cada uma tinha uma pontuação. A contagem final variava de quatro a 20 e então era medido o grau de ansiedade do paciente. Vários autores, em trabalhos posteriores, além de testarem sua confiabilidade e validade, se utilizaram dela em suas pesquisas ou então fizeram uma modificação que permitisse sua aplicação de maneira específica.

Mostrando a importância das escalas, Hosey e Blinkhorn(1995) realizaram um estudo amplo usando quatro delas, (Frankl, Houpt, Visual Analogue e Global Rating) e as testaram quanto ao seu grau de concordância e validade. Após concluírem a investigação sugeriram o uso de questionários para pesquisas com adultos e adolescentes e escalas para crianças menores, devido às limitações de vocabulário, compreensão e desenvolvimento emocional delas. No presente caso a avaliação da ansiedade é amplamente baseada em observação e comportamento, utilizando-se de escalas de classificação. Os autores chegam à indicação da Houpt Scale para uma primeira impressão, e da Frankl Behavioral Scale para selecionar os pacientes.

Dentre os vários artigos sobre questionários analisados, alguns podem ser citados como os de Goodman (1997), Goodman e Scott (1999) e Liddell e Locker (1997). São estudos quantitativos, com a aplicação dos mesmos em pais ou professores, diretamente ou através de mala direta. Foram realizados uma análise detalhada dos resultados e um estudo comparativo, aplicando questionários diferentes nos próprios pacientes e que mostraram dados coincidentes, indicando a validade dos mesmos. 
Houve porém o predomínio das escalas dentre as formas de avaliação estudadas. Estudos, que permitiam desde análises qualitativas até quantitativas, mostraram uma variedade de escalas aplicadas em pré-escolares, escolares e até adolescentes. Normalmente, tinham como característica comum a facilidade de aplicação, a simplicidade, a objetividade. Esta revisão destaca as mais conhecidas e utilizadas, e que também foram várias vezes submetidas a testes de confiabilidade e validade.

Para uma visão mais completa sobre as escalas, é interessante a leitura do trabalho de Parkin (1989) que utilizou a "Child's Dental Anxiety Scale" e o de Greenbaum, Turner, Cook III, e Melamed (1990) que aplicaram 15 itens da "Dental Subscale of the Children's Fear Survey Schedule" em crianças, e depois fizeram sua auto-avaliação através do "Self-Assessment Mannequin" (SAM). Os referidos artigos dão explicações detalhadas das escalas bem como de sua aplicação. Outros autores, como Ten Berge e cols, (1998), também utilizaram uma escala para avaliar medo e ansiedade entre homens e mulheres, e fizeram análises comparativas com relação ao sexo e idade. O item idade não produziu diferenças significativas, mas o item sexo mostrou que mulheres têm mais ansiedade que os homens.

Autores que trabalharam buscando respostas diferentes, porém com o mesmo tipo de instrumento, Quinonez, Santos, Boyar e Cross (1997) e Alwin e cols (1994) utilizaram a Child Manifest Anxiety Scale. Os primeiros pesquisaram crianças entre 2 e 5 anos de idade e concluíram que elas, em geral, são tímidas e jovens demais para cooperar, quando estão na cadeira odontológica. Já Alwin e cols (1994) disseram que a criança que é ansiosa no ambiente odontológico não o é em todas as outras situações.

Escalas de classificação foram desenvolvidas por autores diferentes em épocas diversas. De Fee e Himelstein (1969) elaboraram uma Rating Scale e evidenciaram que os primeiros filhos e os filhos mais velhos eram mais sensíveis à dor e mais medrosos que os outros filhos. Duas escalas foram desenvolvidas por Venham, Gaulin-Kremer, Munster, Bengston-Audia, e Cohan (1980) para avaliar a resposta da criança ao tratamento dental: a "Anxiety Rating Scale" (ARS) e a "Uncooperative Behavior Rating Scale” (UBRS). Estas escalas são ferramen- tas importantes, que permitem a comparação de dados de diversas pesquisas pediátricas. Segundo os autores, o desenvolvimento de técnicas de avaliação confiáveis e válidas é o maior pré-requisito para refinar a pesquisa de comportamento pediátrico e para melhorar o controle/manejo clínico da ansiedade. As escalas são fáceis de administrar e conceituar e, de acordo com estes autores, suas qualidades devem ser:

- Quanto à confiabilidade: uma escala altamente confiável produz resultados similares quando usada para avaliar a mesma amostra de comportamento em tempos diferentes ou por observadores diferentes.

- Quanto à validade: reflete o grau para o qual a escala mede o que é proposto medir. Uma escala válida é aquela que mede com especificidade e acuidade o único traço que foi designado medir.

- Quanto às propriedades mensuráveis: referem-se à natureza das categorias da escala e ao relacionamento entre os pontos da escala. Somente podem ser determinadas através de um estudo empírico da performance da escala em situações de julgamento.

Weinstein, Milgron, Hoskuldsson, Golletz, Jeffcott, e Koday (1996) também foram criadores de uma escala, a Child Dental Control Assessment. Seu artigo descreve desde o estudo piloto até os dois estudos finais definitivos, mostrando porém que os autores ainda têm a pretensão de adicionar novos itens e talvez alterar a forma de aplicação.

\section{Discussão}

Não foram encontrados na literatura instrumentos de avaliação para crianças menores de 3 anos.

A maioria dos pesquisadores trabalhou com faixa etária acima de 3 anos, impedindo que as escalas citadas sejam utilizadas na prática clínica ou em pesquisas, com sujeitos de idade menor. Para que uma escala seja usada em uma faixa etária menor, seriam necessários equipamentos e condutas específicas, uma auxiliar duplamente treinada, sendo que isto somente dificultaria o bom andamento de pesquisas e tornaria necessária a utilização de dois ambientes de trabalho distintos. 
Evidencia-se a necessidade de trabalhar com uma faixa etária baixa, com a finalidade de preencher lacunas existentes na literatura, e por ser esta uma faixa etária difícil que inibe a atuação do odontopediatra (Araújo, 1990).

Também, observou-se que existe uma grande preocupação, entre os diversos autores pesquisados, quanto ao uso de escalas ou questionários como uma forma de avaliação, quantificação ou classificação do medo e ansiedade durante o tratamento odontológico. Porém não se encontrou nenhum autor que estivesse interessado em verificar se havia ou não a presença do medo ou ansiedade frente a uma situação nova. Não se encontraram trabalhos que envolvam uma avaliação inicial dos determinantes do medo na seqüência do atendimento, nem avaliação das reações da criança em uma primeira visita a um ambiente odontológico.

\section{Conclusão}

As escalas estudadas mostraram existir uma lacuna de informações sobre ansiedade e medo na situação odontológica no atendimento de crianças de uma faixa etária mais baixa, não sendo aplicáveis às escalas e questionários existentes em todos os casos nem em todos os pacientes.

Conclui-se que, o odontopediatra não possui uma ferramenta adequada que permita avaliar uma criança em sua primeira visita, e deste modo, saber como proceder ou como se comportar frente a esta criança, no caso de haver necessidade de um tratamento odontológico, ou apenas de condicionamento ao ambiente e equipamento para torná-la um futuro paciente.

Surge assim a idéia de elaborar uma escala que atinja uma faixa etária mais baixa que as encontradas na literatura, que seja de simples aplicação, objetiva em seus resultados e que sua praticidade favoreça o uso, tanto por parte dos profissionais que trabalham com pesquisa, quanto dos que se acham inseridos em consultórios particulares. A proposta é de uma Escala de Observação do Comportamento Infantil.

\section{Referências Bibliográficas}

Alwin, N., Murray, J.J. \& Niven, N. (1994). The effect of children's dental anxiety on the behavior of a dentist. International Journal of Paediatric Dentistry, 4(1), 19-24.

Araújo, G.B.C. (1990). Aspectos psicológicos no atendimento de crianças de um a três anos de idade. Odontólogo Moderno, 17(11/12), 17-20.

Corah, N.L. (1969). Development of a dental anxiety scale. Journal of Dental Research, 48, 596.

De Fee, J.F. \& Himelstein, P. (1969). Children's fear in a dental situation as a function of birth order. The Journal of Genetic Psychology, 115, 253255.

Frankl, S.N., Shiere, F.R. \& Fogels, H.R. (1962). Should the parent remain with the child in the dental operatory? Journal of Dentistry for Children, 29, 150-163.

Goodman, R. (1997). The strengths and difficulties questionnaire: a research note. Journal of Child Psychology and Psychiatry, 38(5), 581-586.

Goodman, R. \& Scott, S. (1999). Comparing the strengths and difficulties questionnaire and the child behavior checklist: is a small beautiful? Journal of Abnormal Child Psychology, 27(1), 17-24.

Greenbaum, P.E., Turner, C., Cook III, E.W. \& Melamed, B.G. (1990). Dentist's voice control: Effects on children's disruptive and affective behavior. Health Psychology, 9(5), 546-558.

Guedes-Pinto, A.C. (1991). Conduta clínica e psicologia em odontologia pediátrica. São Paulo: Santos.

Hosey, M.T. \& Blinkhorn, A.S. (1995). An evaluation of four methods of assessing the behaviour of anxious child dental patients. International Journal of Paediatric Dentistry, 5(2), 87-95.

Liddell, A. \& Locker, D. (1997). Gender and age differences in attitudes to dental pain and dental control._Community Dentistry Oral Epidemiology, 25(4), 314-318.

Parkin, S.F. (1989). Assessment of the clinical validity of a simple scale for rating children's 
dental anxiety.ASDC Journal of Dentistry for Children, 56(1), 40-43.

Quinonez, R., Santos, R.G., Boyar, R. \& Cross, H. (1997). Temperament and trait anxiety as predictors of child behavior prior to general anesthesia for dental surgery. Pediatric Dentistry, 19(6), 427-431.

Silva, H.C., Ammon, I.O.N. \& Vieira R.S. (1994). Adaptação do comportamento de uma criança de 3 anos para o tratamento odontológico. Revista de Odontopediatria, 3(3), 115-123.

Ten Berge, M., Hoogstraten, J., Veerkamp, J.S.J. \& Prins, P.J.M. (1998). The dental subscale of the Children's Fear Survey Schedule: a factor analytic study in the Netherlands. Community Dentistry and Oral Epidemiology, 26(5), 340343.

Venham, L.L., Gaulin-Kremer, E., Munster, E., Bengston-Audia, D. \& Cohan, I. (1980). Interval rating scales for children's dental anxiety and uncooperative behavior. Pediatric Dentistry, 2(3), 195-202.

Weinstein, P., Milgron, P., Hoskuldsson, O., Golletz, D., Jeffcott, E. \& Koday M. (1996). Situation especific child control: a visit to the dentist. Behavior Research and Therapy, 34(1), 11-21.

Winer, G.A. (1982). A review and analysis of children's fearful behavior in dental settings. Child Development, 53(5), 1111-1133. 